M. Koike

Nagoya Math. J.

Vol. 56 (1974), 45-52

\title{
ON SOME $p$-ADIC PROPERTIES OF THE EICHLER-SELBERG TRACE FORMULA
}

\author{
MASAO KOIKE
}

In this paper we shall prove some congruence relations $\bmod p^{\alpha}$ between the traces of Hecke operators $T\left(p^{m}\right)$ which act on the space of cusp forms of different weights satisfying some congruences $\bmod p^{\alpha}-p^{\alpha-1}$. The method of the proof is very simple and is applicable to all the cases where the trace formula for Hecke operators are already known.

As an application, we can obtain the trace formula for $p$-adic Hecke operators $\tilde{U}_{\lambda}\left(p^{m}\right)$ as a $p$-adic limit of the trace of 'classical' Hecke operators. The trace formula for $p$-adic Hecke operators was first given by B. Dwork [1] by using the Monsky trace formula in the case of level 2 , but our method of computation seems to be more elementary.

\section{§ 1. Congruences between the traces of Hecke operators in elliptic modular case}

We shall consider the elliptic modular case in detail, because the essential point of the proof is completely contained in this case. We shall fix a prime number $p$ once and for all. For any positive even integer $k$, let $\varsigma_{k}$ be the space of all modular cusp forms of weight $k$ with respect to $\Gamma=S L(2, Z)$. For each positive integer $n$, let $\Gamma^{(n)}$ be the set of all two by two matrices such that the entries are rational integers and the determinant is $n$, and let

$$
\Gamma^{(n)}=\Sigma_{\lambda} \Gamma\left(\begin{array}{ll}
a_{\lambda}^{(n)}, & b_{\lambda}^{(n)} \\
c_{\lambda}^{(n)}, & d_{\lambda}^{(n)}
\end{array}\right)
$$

be a left $\Gamma$-coset decomposition of $\Gamma^{(n)}$. The Hecke operators $T_{k}(n)$ which act on $\mathfrak{S}_{k}$ as linear endomorphisms are defined by

$$
\widetilde{S}_{k} \ni f(\tau) \mapsto f(\tau) \mid T_{k}(n)=n^{k-1} \Sigma_{\lambda} f\left(\frac{a_{\lambda}^{(n)} \tau+b_{\lambda}^{(n)}}{c_{\lambda}^{(n)} \tau+c_{\lambda}^{(n)}}\right)\left(c_{\lambda}^{(n)} \tau+d_{\lambda}^{(n)}\right)^{-k} .
$$

Received April 6, 1974. 
The Eichler-Selberg trace formula for $T_{k}(n)$ reads as follows:

$$
\begin{aligned}
\operatorname{tr} T_{k}(n)= & \Sigma_{\left\{\rho, \rho^{\prime}\right\}} \Sigma_{\mathrm{o}}-\frac{h_{\mathrm{o}}}{w_{\mathrm{o}}} F^{(k-2)}\left(\rho, \rho^{\prime}\right)-\sum_{d \leq \sqrt{n}}^{\prime} d^{k-1} \\
& +\delta(\sqrt{n}) \frac{k-1}{12} n^{k / 2-1}+ \begin{cases}0, & (k>2), \\
\Sigma_{d \mid n} d, & (k=2),\end{cases}
\end{aligned}
$$

where $F^{(k-2)}\left(\rho, \rho^{\prime}\right)=\rho^{k-1}-\rho^{\prime k-1} / \rho-\rho^{\prime},\left\{\rho, \rho^{\prime}\right\}$ runs over all pairs of mutually conjugate irrational imaginary quadratic integers with norm $n, \mathfrak{o}$ runs over all orders of imaginary quadratic fields such that $\mathfrak{v} \ni \rho$; $w_{0}, h_{0}$ are the number of unity contained in $\mathfrak{0}$, the number of proper o-ideal classes respectively; in the second summand under $\Sigma^{\prime}, d^{k-1}$ should be replaced by $\frac{1}{2} d^{k-1}$ when $d=\sqrt{n}$ is rational, and finally $\delta(\sqrt{n})$ represents 1 resp. 0 when $\sqrt{n}$ is rational resp. irrational.

Now we prove the following proposition.

Proposition 1. Let $\alpha$ be a positive integer, and let $\lambda^{\prime}>\lambda$ be even rational integers which are not smaller than $2 \alpha+2$ for $p \geq 5,2 \alpha+6$ for $p=2$ and $2 \alpha+4$ for $p=3$, and which are congruent to each other $\bmod p^{\alpha}-p^{\alpha-1}$ for $p \geq 5$ and $\bmod p^{\alpha+1}-p^{\alpha}$ for $p=2,3$. Then it holds that

$$
\operatorname{tr} T_{\lambda^{\prime}}\left(p^{m}\right) \equiv \operatorname{tr} T_{\lambda}\left(p^{m}\right) \quad\left(\bmod p^{\alpha}\right)
$$

for any $m>0$.

Proof. For simplicity we prove only for $p \geq 5$. Proofs for other cases are same. We consider the Eichler-Selberg trace formula for $T_{\lambda^{\prime}}\left(p^{m}\right) \bmod p^{\alpha}$. Since $\lambda^{\prime}, \lambda$ are not equal to 2 , the fourth summand is zero. The second resp. third summand is congruent to zero resp. one $\bmod p^{\alpha}$, since $\lambda^{\prime} / 2-1 \geq \alpha$. Let $K$ be an imaginary quadratic field which contains $\left\{\rho, \rho^{\prime}\right\}$, and let $(K / p)$ be Kronecker's symbol. In the case $(K / p)$ $=-1$ or $0, F^{\left(\lambda^{\prime}-2\right)}\left(\rho, \rho^{\prime}\right)$ is easily proved to be congruent to zero $\bmod p^{\alpha}$, since $\lambda^{\prime} / 2-1 \geq \alpha$. We may assume $(K / p)=1, p=\mathfrak{p} \cdot \mathfrak{p}^{\prime}$ with prime ideals $\mathfrak{p} \neq \mathfrak{p}^{\prime}$ in $K$. If $\rho \equiv 0(\bmod p)$, the corresponding term in $(1)$ is congruent to zero $\bmod p^{\alpha}$, so we may assume $\rho \neq \equiv 0(\bmod \mathfrak{p})$. Then we have $\rho^{p^{\alpha}-p^{\alpha-1}} \equiv 1\left(\bmod p^{\alpha}\right)$, so we have $F^{\left(\lambda^{\prime}-2\right)}\left(\rho, \rho^{\prime}\right) \equiv \rho^{\lambda^{\prime}-1} / \rho-\rho^{\prime} \equiv \rho^{\lambda-1} / \rho-\rho^{\prime}$ $\equiv F^{(\lambda-2)}\left(\rho, \rho^{\prime}\right)\left(\bmod \mathfrak{p}^{\alpha}\right)$. For $p \geq 5, w_{0}$ are all $p$-adic units, so the proof is completed.

Q.E.D.

COROLlaRY. Let $\lambda$ be an even integer. Let $\left\{\lambda_{\alpha}\right\}$, for $1 \leq \alpha<\infty$, be 
an increasing sequence of rational integers satisfying that $\lambda_{\alpha}$ is not smaller than $2 \alpha+2$ for $p \geq 5,2 \alpha+6$ for $p=2$, and $2 \alpha+4$ for $p=3$, and that $\lambda_{\alpha}$ is congruent to $\lambda \bmod p^{\alpha}-p^{\alpha-1}$ for $p \geq 5$ and $\bmod p^{\alpha+1}-p^{\alpha}$ for $p=2,3$. Then, for any $m>0$, there exists a p-adic limit of the sequence $\left\{\operatorname{tr} T_{\lambda_{\alpha}}\left(p^{m}\right)\right\}$ and the following formula is valid;

$$
\lim _{\alpha \rightarrow \infty} \operatorname{tr} T_{\lambda_{\alpha}}\left(p^{m}\right)=\Sigma_{\left\{\rho, \rho^{\prime}\right\}} \Sigma-\frac{h_{0}}{w_{0}} \frac{\rho^{\lambda-1}}{\rho-\rho^{\prime}}-1 .
$$

Here the notations are as follows: $\tilde{K}$ is the field generated by all imaginary quadratic fields $K$ such that Kronecker's symbol $(K / p)$ is 1 , and $P$ is a fixed divisor of $\tilde{K}$ extending $p$ of $Q . \quad\left\{\rho, \rho^{\prime}\right\}$ runs over all pairs of mutually conjugate irrational imaginary quadratic integers with no' $m p^{m}$ which are contained in $\tilde{K}$ and $\rho$ is assumed not to be congruent to zero $\bmod P$. $\mathcal{0}$ runs over all orders of imaginary quadratic fields such that o $\ni \rho$.

This follows immediately from the proof of Proposition 1.

\section{§ 2. Congruences between the traces of Hecke operators in more general cases}

2-1. Let $q$ be a positive power of $p$. We note that the essential point of the proof of Proposition 1 is the following properties of the Eichler-Selberg trace formula:

(P-1) The trace of $T_{k}(q)$ (for $k>2$ ) is represented by a sum which runs over an index set depending only on $q$, but not on $k$.

(P-2) Denote by $I(q)$ the common index set in the sense of (P-1). Then $I(q)$ is a finite set and each term, say $a_{k, q}^{(i)}(i \in I(q))$, of the trace is one of the following three types:

(i) $c_{k}^{(i)} \cdot\left(\rho^{k-1}-\rho^{\prime k-1}\right) /\left(\rho-\rho^{\prime}\right)$, where $\rho$ is an algebraic integer whose norm is equal to $q$, and whose degree is 2 (which is a fixed number related only to $\Gamma=S L(2, Z)$ ), and $\rho^{\prime}$ is the complex conjugate of $\rho$, and $c_{k}^{(i)}$ is a rational number depending only on $i$.

(ii) $c_{k}^{(i)} p^{k / 2-1}$, where $c_{k}^{(i)}$ is a rational number whose denominator depends only on $i$.

(iii) $c_{k}^{(i)}$, which is a constant depending only on $i$.

Roughly speaking, when we investigate $c_{k}^{(i)}$ more carefully, we can obtain conditions for weights under which the congruence relation (1) is valid.

2-2. We now consider the trace formula for more general cases. As far as we know, the trace formula for Hecke operators is already 
obtained in the explicit form for the following cases: (1) the congruence subgroup $\Gamma_{0}(N)$ consisting of all two by two integral unimodular matrices $\left(\begin{array}{ll}a & b \\ c & d\end{array}\right)$ such that $c \equiv 0(\bmod N)$, (2) the Fuchsian group of the first kind consisting of all units with norm 1 in a certain order of an indefinite division quaternion algebra over $Q$, (3) the discrete subgroup of finite products of $G L(2, R)$, with the quotient of volume finite, obtained from the group of all units in a maximal order of an indefinite quaternion algebra over a totally real number field. As for the detailed formula we refer to M. Eichler [3] [4], H. Shimizu [11] and H. Hijikata [5]. It should be remarked that the trace formula in [5] and [11] contains the elliptic modular case but the former coincides with (1) up to a factor $n^{1-k / 2}$. Therefore we must multiply some factor on the formulae in [5] and [11] if we consider the congruence relations between the traces. Then, for these three cases, it is immediately checked that these trace formulae also satisfy the above properties (P-1) and (P-2), (although (P-2) must be slightly modified in the case (3)).

2-3. We shall consider the case (2) for example.

Let $A$ be an indefinite division quaternion algebra over $Q$ and let $\subseteq$ be an order of $A$. We put $A_{p}=A \otimes Q_{p}$ (over $Q$ ), and $\bigcirc_{p}=\subseteq \otimes Z_{p}$ (over $Z$ ). Let $F_{1}$ be the product of all primes $p$ for which $A$ is ramified. Take a positive rational integer $F_{2}$ which is prime to $F_{1}$ and is squarefree. Then there exists an order $\mathfrak{D}$ of $A$ such that $\mathfrak{D}_{p}$ is a maximal

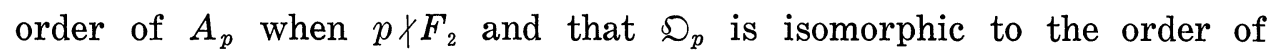
matrices $\left(\begin{array}{ll}a & b \\ p c & d\end{array}\right)$ with $a, b, c, d \in Z_{p}$ when $p \mid F_{2}$. Hereafter we only consider orders $\subseteq$ of this type. Let $\Gamma$ be the group of units of $\subseteq$ with norm 1. Then $\Gamma$ is considered as an Fuchsian group of the first kind when $\Gamma$ is canonically imbedded in $A \otimes_{Q} R$ which is isomorphic to $M(2, R)$. Let $\widetilde{S}_{k}(T)$ be the space of all cusp forms with respect to $\Gamma$ of weight $k$, and let $T_{k}(n)$ be the usual Hecke operators acting on $\widetilde{\varsigma}_{k}(\Gamma)$ as linear endomorphisms.

Eichler's trace formula for $T_{k}\left(p^{m}\right)$ which $p \nmid F_{2}$ and $k>2$ reads as follows :

$$
\operatorname{tr} T_{k}\left(p^{m}\right)=\delta(m) \frac{k-1}{12} p^{m(k / 2-1)} \prod_{\ell \mid F_{1}}(\ell-1) \prod_{\ell \mid F_{2}}(\ell+1)
$$




$$
+\Sigma_{\{0 \ni \rho\}}-\frac{h_{0}}{w_{0}} \prod_{\ell \mid F_{1}}\left(1-\left\{\frac{\mathfrak{D}}{\ell}\right\}\right) \prod_{\ell \mid F_{2}}\left(1+\left\{\frac{\mathfrak{\rho}}{\ell}\right\}\right) F^{(k-2)}\left(\rho, \rho^{\prime}\right)
$$

where $\left\{\rho, \rho^{\prime}\right\}$ and $\mathfrak{o}$ runs over the same set as in (1), and $h_{0}, w_{0}$ are the same as in (1). $\{\mathfrak{o} / \ell\}$ is equal to 1 when the conductor of $\mathfrak{D}$ is divided by $\ell$, and $\{\mathfrak{o} / \ell\}$ is equal to Kronecker's symbol $(Q(\rho) / \ell)$ when the conductor of $\mathfrak{o}$ is prime to $\ell$. $\delta(m)$ is equal to 1 resp. 0 when $m$ is even resp. odd.

By the consideration in 2-2 and the proof of Proposition 1 the following is easily proved:

Proposition 2. Let $p$ be a prime number such that $p \nmid F_{2}$. Let $\alpha$ be a positive integer, and let $\lambda^{\prime}>\lambda$ be even integers which are not smaller than $2 \alpha+2$ for $p \geq 5,2 \alpha+6$ for $p-2$ and $2 \alpha+4$ for $p=3$, and which are congruent to each other $\bmod p^{\alpha}-p^{\alpha-1}$ for $p \geq 5$ and $\bmod p^{\alpha+1}$ $-p^{\alpha}$ for $p=2,3$. Then it holds that

$$
\operatorname{tr} T_{\lambda^{\prime}}\left(p^{m}\right) \equiv \operatorname{tr} T_{\lambda}\left(p^{m}\right) \quad\left(\bmod p^{\alpha}\right)
$$

for any $m>0$.

\section{§3. Trace formula for $\tilde{U}_{k}(p)^{m}$}

3-1. We shall show that the result in $\S 1$ gives a trace formula for $p$-adic Hecke operator $\tilde{U}_{2}(p)^{m}$. As for the definitions and notations, we refer to [7]. Let $\mathscr{S}^{(\lambda)}$ be the space of all $p$-adic cusp forms of weight $\lambda$, and let $\tilde{U}_{\lambda}(p)$ be the $p$-adic Hecke operator on $\mathscr{S}^{(\lambda)}$. For $\alpha>0$, put $\lambda_{\alpha}=\lambda+p^{\alpha}-p^{\alpha-1}$, and let $\left\{F_{\alpha, i}^{(\lambda)}\right\}, 1 \leq i \leq d_{\alpha}^{(\lambda)}$ be the compatible system of free basis of $\widetilde{S}_{\lambda_{\alpha}, \mathrm{O}_{T}}$. Put $\tilde{F}_{i}^{(\alpha)}=\lim _{\alpha \rightarrow \infty} F_{\alpha, i}^{(\lambda)}$, then $\left\{\tilde{F}_{i}^{(\lambda)}\right\}, 1 \leq i \leq \infty$, forms an orthonormal basis of $\mathscr{S}^{(\lambda)}$ over $Q_{p}^{\infty}$. Put $\tilde{F}_{i}^{(\lambda)} \mid \tilde{U}_{2}(p)^{m}=\sum_{i=1}^{\infty} a_{i j}(m) \tilde{F}_{j}^{(\lambda)}$, with $a_{i j}(m) \in \mathfrak{D}_{p}^{\infty}$. Let $\left\{e_{i}\right\}, 1 \leq i \leq \infty$, be a sequence of positive powers of $p$. Then, it is proved that $\tilde{U}_{\lambda}(p)$ is completely continuous on the $p$ adic Banach space $\mathscr{S}^{(\lambda)}$ with the orthonormal basis $\left\{e_{i} \tilde{F}_{i}^{(\lambda)}\right\}$ for suitable $\left\{e_{i}\right\}$. Therefore $\sum_{i=1}^{\infty} a_{i j}(m)$ converges, and we define $\operatorname{tr} \tilde{U}_{\lambda}(p)^{m}=\sum_{i=1}^{\infty} a_{i j}(m)$, which is proved to be independent of the choice of $\left\{F_{\alpha, i}^{(\lambda)}\right\}$.

THEOREM 1. The following formula is valid;

$$
\operatorname{tr} \tilde{U}_{\lambda}(p)^{m}=\Sigma_{\left\{\rho, \rho^{\prime}\right\}} \Sigma_{0 \ni \rho}-\frac{h_{0}}{w_{0}} \frac{\rho^{\lambda-1}}{\rho-\rho^{\prime}}-1,
$$

where the right hand side is the same as in (3). 
Proof. We can take $\alpha_{0}$ such that $\lambda_{\alpha} \geq 2 \alpha+6$ for any $\alpha \geq \alpha_{0}$. By the recursion formula for $T_{k}(n)$ and the definition of $\tilde{U}_{\lambda}(p)$, we have

$$
\begin{aligned}
F_{\alpha, i}^{(\lambda)} \mid T_{\lambda_{\alpha}}\left(p^{m}\right) & \equiv F_{\alpha, i}^{(\lambda)} \mid T_{\lambda_{\alpha}}(p)^{m} & & \left(\bmod \widetilde{\mathfrak{P}}^{\alpha}\right) \\
& \equiv \tilde{F}_{i}^{(\lambda)} \mid \tilde{U}_{\lambda}(p)^{m} & & \left(\bmod \widetilde{\mathfrak{B}}^{\alpha}\right),
\end{aligned}
$$

for $\alpha \geq \alpha_{0}$. For this and Proposition 1, it follows that

$$
\operatorname{tr} T_{\lambda_{\alpha+1}}\left(p^{m}\right) \equiv \operatorname{tr} \tilde{U}_{\lambda}(p)^{m} \quad\left(\bmod \tilde{\mathfrak{B}}^{\alpha}\right),
$$

therefore we obtain (4) by Corollary of Proposition 1.

Q.E.D.

3-2. (Fredholm determinant and Hecke polynomials.) We shall give here a relation between the Fredholm determinants of the $p$-adic Hecke operators and the Hecke polynomials in elliptic modular case. In the case of principal congruence subgroup of level 2 of the elliptic modular group, B. Dwok [1] gave essentially a relation between the Fredholm determinants of the $p$-adic Hecke operators and some infinite products, and Y. Morita [9] gave a relation between some infinite product slightly different from the above and the Hecke polynomials. Combining these two results together, we could get a desired relation in this case. But this process is complicated. Here, using the $p$-adic trace formula for $\tilde{U}_{k}(p)^{m}$, we shall obtain the relation more directly and explicitly. We shall often refer to Y. Ihara [6] and Y. Morita [8], and use the same notations as in them.

Let $D_{k}^{(p)}(u)=\exp \left\{-\sum_{m=1}^{\infty} \frac{\operatorname{tr} \tilde{U}_{k}(p)^{m}}{m} u^{m}\right\}$ be the Fredholm determinant of $\tilde{U}_{k}(p)$. As is known, $D_{k}^{(p)}(u)$ is a $p$-adic entire function. Let $H_{k}^{(p)}(u)$ $=\operatorname{det}\left(I-T_{k}(p) u+p^{k-1} I u^{2}\right)$ be the Hecke polynomial where $I$ is the identity operator on $\mathfrak{S}_{k}$.

THEOREM 2. Let $k$ be an even positive rational integer. Then it holds that

$$
\begin{aligned}
& D_{k}^{(p)}(u) / D_{2-k}^{(p)}\left(p^{k-1} u\right) \\
& \quad=H_{k}^{(p)}(u)\left(1-\mathrm{p}^{k-1} u\right)\left(1-p^{(k-2) / 2} u\right)^{a+}\left(1+p^{(k-2) / 2} u\right)^{a_{-}} d(t)
\end{aligned}
$$

where

$$
d(t)= \begin{cases}(1-t)^{-1}(1-p t)^{-1}, & k=2 \\ 1, & k>2\end{cases}
$$


The notations are as follows: when $p \geq 5$, we put $a_{+}=\frac{1}{2}\{(k-1)(H-\Delta)$ $\left.+d_{k}+(-1)^{(k-2) / 2} h^{\prime}\right\}$ and $\left.a_{-}=\frac{1}{2}\{k-1)(H-\Delta)+d_{k}-(-1)^{(k-2) / 2} h^{\prime}\right\}$ where $H, h^{\prime}$ and $\Delta$ are as defined by (7), (8), and (45') in [6] and $d_{k}$ is equal to $c_{k}$ defined by (45) in [6] with even $m$.

When $p=2,3$, pe put $a_{+}=\frac{1}{2}\{c(k, 2)+c(k, 1)\}$ and $a_{-}=\frac{1}{2}\{c(k, 1)-$ $c(k, 2)\}$ where $c(k, 1)$ and $c(k, 2)$ are as defined by (13) in [8].

Proof. For simplicity, we prove only for $p \geq 5$. Proofs for other cases are similar. The condition that the mutually conjugate irrational imaginary quadratic integer pair $\left\{\rho, \rho^{\prime}\right\}$ with norm $p^{m}$ appears in the summation of the right hand side of the equation (4) is easily seen to be equivalent to that $\rho$ is not equal to $\pm \sqrt{-p}$ and is primitive, namely, $p^{-1} \rho$ is not an algebraic integer. Therefore, by Lemmas 6 and 8 in [6] with the fact $\rho \rho^{\prime}=p^{m}$, we get the following:

$$
\begin{aligned}
\operatorname{tr} \tilde{U}_{k}(p)^{m}-p^{m(k-1)} \operatorname{tr} \tilde{U}_{2-k}(p)^{m} \\
=\operatorname{tr} U_{k}\left(p^{m}\right)+p^{m(k-1)}+d_{k} p^{m / 2(k-2)} \\
+ \begin{cases}(k-1)(H-\Delta) p^{m / 2(k-2)} & (m: \text { even }) \\
\left(h^{\prime}-\Delta\right)(-p)^{m / 2(k-2)} & (m: \text { odd })\end{cases} \\
- \begin{cases}1+p^{m}, & k=2 \\
0, & k>2 .\end{cases}
\end{aligned}
$$

From this, (5) follows by some calculations.

Q.E.D.

REMARK. For $p=2,3$, it is remarked in [8] that $a_{+}$and $a_{-}$are integers. For $p \geq 5$, it is easily seen that $d_{k}+\Delta$ and $H+h^{\prime}$ are even integers, so $a_{+}$and $a_{-}$are integers.

As for the values of $a_{+}$and $a_{-}$, we have the following proposition:

Proposition 3. For $k>2, a_{+}$(resp. $a_{-}$) is equal to the number of normalized new forms of weight $k$ on $\Gamma_{0}(p)$ whose eigenvalue of $U(p)$ is equal to $p^{(k-2) / 2}$ (resp. $\left.-p^{(k-2) / 2}\right)$. For $k=2, a_{+}-1$ (resp. $a_{-}$) is equal to the number of normalized new forms of weight 2 on $\Gamma_{0}(p)$ whose eigenvalue of $U(p)$ is equal to $p$ (resp. $-p)$.

Proof. Put $b_{+}$(resp. $b_{-}$) the number of normalized new forms of weight $k$ on $\Gamma_{0}(p)$ whose eigenvalue of $U(p)$ is equal to $p^{(k-2) / 2}$ (resp. $\left.-p^{(k-2) / 2}\right)$. By Atkin and Lehner [12], $b_{+}$(resp. $b_{-}$) is equal to the number of normalized new forms of weight $k$ on $\Gamma_{0}(p)$ which are not (resp. are) 
cusp forms with respect to $\Gamma^{*}(p)$, the Fuchsian group of the first kind generated by $\Gamma_{0}(p)$ and $\left[\begin{array}{rr}0 & 1 \\ -p & 0\end{array}\right]$. Let $e_{k}$ and $e_{k}^{*}$ be dimensions of the space of cusp forms of weight $k$ with respect to $\Gamma_{0}(p)$ and $\Gamma^{*}(p)$ respectively. Then the following equalities hold: $e_{k}-e_{k}^{*}=b_{+}+\operatorname{dim} \varsigma_{k}, e_{k}^{*}$ $=b_{-}+\operatorname{dim} \Im_{k}$. On the other hand, $e_{k}$ and $e_{k}^{*}$ are directly computed by the result of Fricke, so we have the following equalities:

$$
\begin{aligned}
& e_{k}-e_{k}^{*}= \begin{cases}a_{+}+\operatorname{dim} \Im_{k}, & k>2, \\
a_{+}-1, & k=2,\end{cases} \\
& e_{k}^{*}=a_{-}+\operatorname{dim} \Im_{k} .
\end{aligned}
$$

Hence Proposition 3 is proved.

Q.E.D.

\section{REFERENCES}

[1] B. Dwork, On Hecke polynomials, Inventiones Math., 12 (1971), 249-256.

[2] B. Dwork, The $U_{p}$ operator of Atkin on modular functions of level 2 with growth conditions, Modular functions of one variable III, lecture note in mathematics, Springer, Berlin-Heidelberg New York, 1973.

[ 3 ] M. Eichler, Zur Zahlentheorie der Quaternionen-Algebren, J. Reine Angew. Math., 195 (1956), 127-151.

[ 4 ] M. Eichler, Eine Verallgemeinerung der Abelschen Integrale, Math. Z., 67 (1957), 217-239.

[ 5 ] H. Hijikata, Explicit formula of the traces of Hecke operators for $\Gamma_{0}(N)$, J. Math. Soc. of Japan, 26 (1974), 56-82.

[6] Y. Ihara, Hecke polynomials as congruence $\zeta$ functions in elliptic modular case, Ann. of Math., 85 (1967), 267-295.

[ 7 ] M. Koike, Congruences between modular forms and functions and applications to the conjecture of Atkin, J. Fac. Sci. Univ. Tokyo, Sec. IA, 20 (1973), 129-169.

[ 8 ] Y. Morita, Hecke polynomials $H_{k}^{(p)}(u)(p=2$ or 3), J. Fac. Sci. Univ. Tokyo, Sec. I., 15 (1968) , 99-105.

[ 9 ] Y. Morita, Hecke polynomials of modular groups, J. Math. Soc. Japan, 21 (1969), 607-637.

[10] J.-P. Serre, Formes modulaires et fonctions zeta p-adiques, Modular functions of one variable III, lecture note in mathematics, Springer, Berlin-Heidelberg-New York, 1973.

[11] H. Shimizu, On traces of Hecke operators, J. Fac. Sci. Univ. Tokyo, Sec. IA, 10 (1963), 1-19.

[12] A. O. L. Atkin, and J. Lehner, Hecke operators on $\Gamma_{0}(m)$, Math. Ann., 185 (1970), 134-160.

Nagoya University 